\title{
1 Allelic variants of the NLR protein Rpi-chc1 differentially 2 recognise members of the Phytophthora infestans 3 PexRD12/31 effector superfamily through the leucine- 4 rich repeat domain
}

5

6

Daniel Monino-Lopez ${ }^{1}$, Maarten Nijenhuis ${ }^{1,2}$, Linda Kodde ${ }^{1}$, Sophien Kamoun ${ }^{3}$, Hamed Salehian ${ }^{1}$, Kyrylo Schentsnyi ${ }^{1,5}$, Remco Stam ${ }^{1,6}$, Anoma Lokossou ${ }^{1}$, Ahmed Abd-El-Haliem ${ }_{1,8}$, Richard GF Visser ${ }^{1}$, Jack H Vossen ${ }^{1}{ }^{*}$

${ }^{1}$ Plant Breeding, Wageningen University \& Research, Droevendaalsesteeg 1, 6708PB, Wageningen, The Netherlands.

${ }_{2}^{2}$ Current address: Agrico Research, Burchtweg 17, 8314PP, Bant, The Netherlands;

${ }^{3}$ The Sainsbury Laboratory, University of East Anglia, Norwich Research Park, Norwich, United Kingdom.

${ }^{5}$ Current address: Center for Plant Molecular Biology, Auf der Morgenstelle 32, 2076

Tübingen, Germany;

${ }^{6}$ Current address: Technical University Munich;

${ }^{8}$ Current address: Rijk Zwaan Breeding B.V., Burgemeester Crezéelaan 40, The Netherlands;

${ }^{*}$ Corresponding author:

Jack H. Vossen

+31317481590

jack.vossen@wur.nl

Word count: Total: 6,301 words; Summary: 200 words; Introduction: 854 words; Materials and Methods: 1,284 words; Results: 2,543 words; Discussion: 1,612 words.

Number of figures: 6 (all in colour)

Number of Tables: 0

Number of supplementary Figures: 5 (all in colour)

Number of supplementary Tables: 7 


\section{Summary}

- Phytophthora infestans is a pathogenic oomycete that causes the infamous potato late blight disease. Resistance $(R)$ genes from diverse Solanum species encode intracellular receptors that recognize $P$. infestans RXLR effector proteins and provide effective defence responses. To deploy these $R$ genes in a durable fashion in agriculture, we need to understand the mechanism of effector recognition and the way the pathogen evades recognition.

- We cloned sixteen allelic variants of the Rpi-chc1 gene from Solanum chacoense and other Solanum species, and identified the cognate $P$. infestans RXLR effectors. These tools were used to study receptor-ligand interactions and coevolution.

- Functional and non-functional alleles of Rpi-chc1 encode Coiled-Coil-Nucleotide Binding-Leucine-Rich-Repeat (CNL) proteins. Rpi-chc1.1 recognised multiple PexRD12 (AVRchc1.1) proteins while Rpi-chc1.2 recognised multiple PexRD31 (AVRchc1.2) proteins, both from the PexRD12/31 superfamily. Domain swaps between Rpi-chc1.1 and Rpi-chc1.2 revealed that overlapping subdomains in the LRR were responsible for the difference in effector recognition.

- This study showed that Rpi-chc1.1 and Rpi-chc1.2, evolved to recognize distinct members of the same PexRD12/31 effector family via the LRR domain. The biased distribution of polymorphisms suggests that exchange of LRRs during host-pathogen co-evolution can lead to novel recognition specificities. These insights will help future strategies to breed for durable resistant varieties.

Key words: NLR cluster, Leucine rich repeat Phytophthora infestans, Late blight resistance gene, RXLR effector, Solanum species, potato 
66

67

68

69

70

\section{Introduction}

Potato (Solanum tuberosum) is the fourth largest food crop in the world after maize, rice and wheat, with more than 368 million tonnes produced in 2018 (FAO, 2020). Potato late blight, caused by the oomycete Phytophthora infestans ( $P$. infestans), is one of the most infamous potato diseases. During the mid-1840s, this pathogen caused the Great Irish Famine from which around one million people died (Callaway, 2013). Nowadays, losses from late blight are estimated to still reach $16 \%$ of the world production and the main disease management is based on biocide applications. Including yield losses and crop protection measures, late blight causes a global economic loss of $€ 5.2$ billion per year (Haverkort et al., 2016).

$P$. infestans is an oomycete with sexual and asexual life cycles, which exhibits a hemibiotrophic lifestyle on potato. Together with its large and fast evolving genome, it leads to the regular emergence of new aggressive and virulent strains. The infection starts when a spore lands on the plant surface, germinates and forms a penetration structure called appressorium. Alternatively, spores can also enter through natural openings such as stomata. After passing the epidermis, hyphae spread intercellularly projecting haustorium structures into the mesophyll cells. These haustoria are specialised infection structures that create an intimate association with the host cell facilitating nutrient uptake, and both apoplastic and cytoplasmic effector secretion (Fry, 2008). Effectors are pathogen molecules that interact with different host targets to suppress the host defence response and enable colonisation. The publication of the $P$. infestans T30-4 genome, revealed the presence of 563 effector genes encoding the conserved Arg-any amino acid-Leu-Arg (RXLR) peptide motif (Haas et al., 2009). These effectors, rapidly evolve by gaining and losing repeat-rich domains through recombination with different paralogs, transposon movement, and point mutations (Goss et al., 2013). During co-evolution, potato has evolved receptors to recognise some of these effectors and trigger an immune response.

Wild Solanum species are the main source of resistance $(R)$ genes to $P$. infestans (Rpi). To date, over 20 Rpi genes have been characterised in different Solanum species, e.g. $R 1, R 2, R 3 a, R 3 b, R 8, R 9 a$ from $S$. demissum, Rpi-blb1, 2 from $S$. bulbocastanum, Rpi-vnt1 from $S$. venturii and Rpi-amr1 from $S$. americanum 
99 (Ballvora et al., 2002; van der Vossen et al., 2003, 2005; Huang et al., 2005; Pel et

100 al., 2009; Lokossou et al., 2009; Li et al., 2011; Jo et al., 2015; Vossen et al., 2016;

101 Witek et al., 2020). All these receptors belong to the nucleotide-binding (NB)-leucine-

102 rich repeat (NLR) type of receptors and contain a coiled-coil domain (CC) in their $\mathrm{N}$ -

103 termini, referred to as CC-NB-LRR or CNL. The recognition of a specific effector or a-

104 virulence factor (AVR) leads to the activation of the plant defences and the restriction

105 of the pathogen growth. To keep up with the fast evolution of effectors, NLR genes

106 are also very diverse and rapidly evolving. Gene duplications, recombinations,

107 unequal crossing overs and transpositions have been proposed to provide the basis

108 for the evolution of the NLR recognition spectrum (Leister, 2004; Mcdowell \& Simon,

109 2006). This fast evolution can lead to the independent development of new Rpi

110 receptors in different geographical locations that recognise the same effector. For

111 instance, the recognition of the effector AVR2 from $P$. infestans, by the unrelated R2

112 and Rpi-mcq1 CNLs (Aguilera-Galvez et al., 2018). R2 is located on chromosome IV

113 in the Mexican species S. demissum, while Rpi-mcq1 is located on chromosome IX

114 from a Peruvian accession of S. mochiquense (Smilde et al., 2005; Foster et al.,

115 2009). When the doubled-monoploid DM1-3 519 R44 potato genome was published,

116755 NLR genes were identified (Jupe et al., 2013). Many of them were found in

117 clusters together with closely related paralogs. All of these clusters were formed in

118 ancestral species and had sequence homology to syntenic genomic regions from

119 other Solanum species harbouring late blight resistance genes. Thus, inactive Rpi

120 homologs (rpi) can be found in all Solanum genomes.

122 Here, we studied Solanum chacoense (S. chacoense); a diploid wild potato relative

123 from South America considered a source of resistance to $P$. infestans. We identified

124 two functionally distinct receptors, Rpi-chc1.1 and Rpi-chc1.2, which are allelic

125 variants that recognise distinct $P$. infestans effectors from the same PexRD12/31

126 effector superfamily. Remarkably, only Rpi-chc1.1 is able to provide resistance

127 against current $P$. infestans isolates. The expression and recognition of PexRD12

128 effectors was associated with Rpi-chc1.1 mediated resistance and, therefore,

129 designated as AVRchc1.1 effectors. PexRD31 effectors were still expressed in

130 current $P$. infestans isolates, but were rapidly downregulated during the interaction

131 with potato. This, potentially explains the inability of Rpi-chc1.2 to provide late blight

132 resistance. We postulate that $R$ pi-chc1.2 is a ubiquitous ancient $R$ gene that was 
133 recently overcome and PexRD31 may have functioned as AVRchc1.2. An allele

134 mining strategy revealed Rpi-chc1 orthologs in different wild Solanum accessions

135 and potato cultivars that could be classified by their sequence and recognition

136 spectrum of AVRchc1.1, AVRchc1.2, or non-functionality. Finally, using domain

137 swaps, we found that the LRR domain harboured the recognition specificity of both

138 AVRchc1.1 and AVRchc1.2. The specificities resided in overlapping LRR

139 subdomains and could not be combined into one active protein using domain

140 exchanges.

\section{Materials and Methods}

143 Plant materials and growth conditions

144 The wild Solanum species used in this study are listed in Table S1 (Tan et al., 2010;

145 Vleeshouwers et al., 2011a). The potato plants were maintained in vitro on MS20 at $14624^{\circ} \mathrm{C}$ under 16/8h day/night regime (Domazakis et al., 2017). The $7650 \mathrm{~F} 1$ population 147 was generated by crossing S. chachoense (CHC543-5) x S. chacoense (CHC544-5).

148 S. tuberosum cv. 'Désirée' was used for stable transformations of the different Rpi149 chc1.1 candidates. Four week old Nicotiana benthamiana leaves were used for 150 agroinfiltration. The agroinfiltrated plants were kept in climate regulated greenhouse 151 compartments of Unifarm (Wageningen University \& Research) at $20-25^{\circ} \mathrm{C}$ and under 152 16/8h day/night regime.

153

\section{BAC clone isolation and sequencing}

156 The procedure has been described in patent US9551007B2. Briefly: Two different

157 BAC libraries were produced using partial digestion of CHC543-5 genomic DNA with 158 HindIII. Fragments larger than 100kb were ligated into pBeloBAC or pCC1BAC arms 159 (Epicenter). The BAC clones were collected and stored as bacterial pools of 160 approximatively 700 to 1000 white colonies. BAC pools were screened with selected 161 markers and individual clones were identified using colony PCR. The ends of positive 162 individual BACs were sequenced for the purpose of fine mapping RH106G03T and 163 RH137D14_C37-7-4. The complete inserts were sequenced using shotgun 164 sequencing of $2 \mathrm{~kb}$ library fragments generated by partial digestion with EcoR 1 by 
165 Macrogen, Inc (Seoul, South Korea). Assembly of the sequences resulted in contigs

166 as indicated in Fig. 1 (Genbank accession number MW383255).

\section{Cloning of Rpi-chc1 allelic variants and chimeric constructs}

The Rpi-chc1 allelic variants were amplified using genomic DNA from the different wild Solanum species using PCR primers as described in Table $\mathbf{S 2}$ and DNA polymerase with proofreading activity. The fragments were cloned into pGEM-T easy vector (Promega) for sequencing. Genbank submission numbers as in Table S1. The Rpi-ber1.1 and Rpi-tar1.1 genes were amplified using primers in the promotor and terminator. The resulting PCR fragments were cloned into pBINPLUS-PASSA (Jo et al., 2016) and were expressed in transgenic Desiree plants under the control of their native regulatory elements. For transient expression analyses, the coding sequences of the allelic variants were cloned under the Rpi-chc1.1 regulatory elements $(900 \mathrm{bp}$ promotor and 400bp terminator) into pDEST using a multisite gateway protocol. Escherichia coli strain $\mathrm{DH} 10 \mathrm{~B}$ was transformed with the gateway reaction products and clones with the correct insert were selected. Agrobacterium tumefaciens AGL1+VirG was used for transient and stable transformations of $N$. benthamiana leaves and S. tuberosum cv. 'Désirée'.

The chimeric constructs were cloned using the Golden Gate modular cloning principle. As acceptor vector, we used a Golden Gate compatible version of pBINPLUS (McBride \& Summerfelt, 1990), PBINPLUS-GG (Vossenberg et al., 2019). The final acceptor vector was constructed to contain $800 \mathrm{bp}$ Rpi-chc1.1 promoter::CDS::1000bp Rpi-ber terminator (Fig. S1). The different PCR fragments were amplified using the Phusion High-Fidelity PCR Kit (Thermo SCIENTIFIC) and primers with Bsal sites as overhang (Table S2) and purified using the DNA

192 Clean\&Concentrator Kit (ZYMO RESEARCH). PCR fragments and the acceptor

193 vector were incubated in Buffer G (Thermo SCIENTIFIC) with ATP 1mM for thirty cycles of $37^{\circ} \mathrm{C}$ for $5 \mathrm{~min}+16^{\circ} \mathrm{C}$ for $5 \mathrm{~min}$. Additionally, we performed a final step at $37^{\circ}$ for $10 \mathrm{~min}$, to digest the plasmids wrongly assembled, and $65^{\circ} \mathrm{C}$ for $20 \mathrm{~min}$, to heat inactivate the Bsal enzyme. 
200 Transient expression of the different receptors and PexRD12/31 effectors were

201 performed in four weeks old $N$. benthamiana leaves. R3a/AVR3a was used as a 202 positive control. All the constructs were agroinfiltrated at an $\mathrm{OD}_{600}$ of 0.5 . Each 203 construct was agroinfiltrated twice on two leaves of four plants in at least two 204 independent experiments. Cell death responses were observed after 3-4 days post 205 inoculation.

Phylogenetic analysis of Rpi-chc1.1 homologs and the PexRD12/31 superfamily

210 The sequences of the PexRD12/31 effectors were retrieved from the $P$. infestans

211 T30-4 genome (Haas et al., 2009). Twenty family members were found to form the

212 PexRD12/31 superfamily. The coding sequences of the Rpi-chc1 variants as 213 obtained in this study, were aligned using MUSCLE and a neighbour joining tree was 214 calculated using Megalign from the DNAstar package. The closest homolog of Rpi215 chc1.1 from the DM reference genome (SoltuDM10G021850.1) was used as an 216 outgroup.

217 The protein sequences of PexRD12/31 effectors were aligned using Clustal OMEGA 218 and manually edited in MEGAX (Sievers et al., 2011; Kumar et al., 2018). The 219 phylogenetic relationship was inferred using the Maximum Likelihood method based 220 on the JTT matrix-based model in MEGAX with 1000 bootstraps (Jones et al., 1992).

221 The tree with the highest log likelihood was shown. The two more distant effectors 222 PITG_16428 and PITG_09577, served as an outgroup.

\section{P. infestans isolates and Detach Leaf Assay (DLA)}

226 The $P$. infestans isolates used in this study (90128, IPO-C and NL08645) were 227 retrieved from our in-house collection. Isolates were grown at $15^{\circ} \mathrm{C}$ on solid rye 228 medium in the dark (Caten \& Jinks, 1968). After two weeks, sporulating mycelium 229 was flooded with $20 \mathrm{~mL}$ of ice-cold water, adjusted to 70 zoospores/ $\mu \mathrm{L}$ and incubated 230 at $4^{\circ} \mathrm{C}$ for $2-3$ hours. After the incubation, the detached leaves were inoculated with $23110 \mu \mathrm{L}$ of the zoospore suspension on the abaxial side of the leaves. Detached leaves 232 were inserted into wet floral foam. For each biological replicate the three leaflets from 
233 four leaves from two independent plants were used. Twelve spots on each leaf were

234 inoculated with the zoospore suspension and closed in a plastic bag, to maintain high

235 humidity. The leaves were kept in a climate cell at $18^{\circ} \mathrm{C}$ for 5 days. Disease

236 resistance was scored on a scale from 1 to 10 for each leaflet. 10=no symptoms;

$2379=\mathrm{HR}$ no larger than the inoculum droplet; 8=HR lesion of up to $0,5 \mathrm{~cm}$ diameter;

2387 =diffuse lesions up to $1 \mathrm{~cm}$ diameter, no sporulation, no water soaking; $5=$ lesions

239 larger than $1 \mathrm{~cm}$ sometimes with water soaking, no sporulation; 4=large water

240 soaked lesions with sporulation only visible through binoculars; $2=$ large lesions with

241 macroscopically visible sporulation on one side of the leaflet; $1=$ large lesions with

242 macroscopically visible sporulation on both sides of the leaflet.

\section{Relative effector and $\boldsymbol{R}$ gene expression}

246 The $P$. infestans effectors used in this study are listed in Table S3. The different 247 genotypes were inoculated with the different $P$. infestans isolates and samples were 248 collected after 0, 3, 8, 24, 48, 72, 96 and 120 hours. Infected plant material with the 249 different $P$. infestans isolates was collected and RNA was isolated using RNA 250 Purification Kit (QIAGEN). The isolated RNA was converted into cDNA using the 251 QuantiTect Reverse Transcription Kit (QIAGEN). The primers used in this study are 252 listed in Table S2. The expression of the different effectors in the infected material 253 was evaluated using RT-qPCR SYBR Green (Bio-Rad). The samples were heated to $2549^{\circ} \mathrm{C}$ for $2 \mathrm{~min}$. Then 40 cycles of $15 \mathrm{sec}$ at $95^{\circ} \mathrm{C}, 30 \mathrm{sec}$ at $60^{\circ} \mathrm{C}$ and $30 \mathrm{sec}^{\circ} 72^{\circ} \mathrm{C}$. 255 Fluorescence was measured after each cycle. After the final amplification cycle a 256 melting curve was calculated. Relative gene expression was calculated using the 2 $257 \triangle \Delta C T$ method (Livak \& Schmittgen, 2001). The normalised gene expression was 258 obtained by dividing the relative gene expression by the relative $P$. infestans 259 elongation factor 2 gene (ef2) expression.

260

sgRNA and CRISPR-Cas9 construct design

262

263 The CRISPOR web tool (http://crispor.org) was used to design the sgRNAs with 264 lower off-target and higher on-target potentials (Concordet \& Haeussler, 2018). 
266 A Modular Cloning (MoClo) system based on the Golden Gate cloning technology

267 was used to assemble the different sgRNAs and binary vectors as previously

268 described for tomato mutagenesis (Engler et al., 2008; Weber et al., 2011). Briefly,

269 each sgRNA was fused to the Arabidopsis thaliana U6-26 promoter as AtU6-

270 26::gRNA. The Level 1 constructs plCH47732-pNOS::NPTII::tOCS, plCH47742-

271 p2x35S::hCas9::tNOS and the linker p/CH41780 were used to build the Level 2

272 vector pICSL4723 (Werner et al., 2012). The primers used for cloning the gRNAs are

273 listed in Table S2.

274

275 Results

276 Cloning and characterization of Rpi-chc1.1

277

278 The S. chacoense accession CHC543 from Bolivia is a previously described wild 279 potato relative harbouring resistance to $P$. infestans (Vleeshouwers et al., 2011a). To 280 identify the genetic locus of resistance, the resistant seedling CHC543-5 was crossed 281 with the susceptible seedling CHC544-5 to generate the F1 population 7650, 282 consisting initially of 212 individuals. This population was challenged with $P$. infestans 283 isolate 90128 in a detached leaf assay (DLA). A clear 1:1 segregation was observed, 284 indicating the presence of a single dominant resistance gene which will henceforth be 285 referred to as Rpi-chc1. CAPS markers from chromosome 10 were tested as this 286 chromosome was known to harbour Rpi-ber from the related species $S$. berthaultii 287 (Vossen et al., 2013). The marker TG63 in chromosome 10 was indeed linked to the 288 Rpi-chc1 resistance. Successive fine mapping in a recombinant population 289 representing 2357 individuals was performed using markers derived from RH89-39-

29016 BAC clones from chromosome 10 (PGSC) (The Potato Genome Sequencing 291 Consortium, 2011; Sharma et al., 2013). A narrow genetic window between markers 292 RH106G03-T and RH97D21_C21-4 was identified to contain Rpi-chc1 (Fig. 1a). To 293 generate a physical map of the mapping interval, two Bacterial Artificial Chromosome 294 (BAC) clones, B1 and B2, were selected from a BAC library that was derived from 295 CHC543-5 genomic DNA. After sequencing the BAC clones, two NLR genes were 296 identified in clone B1 and another six NLR in clone B2. Further fine-mapping revealed 297 that only the last six were located within the mapping interval and only three (B2-1, 298 B2-2 and B2-3) encoded complete NLR proteins (Fig. 1b). The three candidates were 
299 subcloned including their native 5' and 3' regulatory elements, and complementation

300 analyses were performed in $N$. benthamiana. After two days, the agroinfiltrated area

301 was challenged with $P$. infestans 90128. Rpi-blb1, which was shown to provide

302 resistance to $P$. infestans, was used as a positive control. The leaves agroinfiltrated

303 with candidate B2-3 and Rpi-blb1 showed severely compromised pathogen growth,

304 while leaves with candidates B2-1 and B2-2 were completely susceptible to $P$.

305 infestans 90128 (Fig. S2). This result suggested that B2-3 was the gene in CHC543-

3065 that provides resistance to $P$. infestans. To verify this result, the three candidates

307 B2-1, B2-2 and B2-3 were stably transformed into the susceptible S. tuberosum cv.

308 'Désirée'. Indeed, only the events containing candidate B2-3 showed resistance to $P$.

309 infestans (Fig. 1c). Furthermore, we specifically targeted the B2-3 candidate with

310 sgRNAs and CRISPR-Cas9 enzyme by stable transformation of the resistant

311 CHC543-5 genotype with. The transformation events were challenged with $P$.

312 infestans 90128 and IPO-C isolates, and $48 \%$ of the transformants became

313 susceptible to both isolates (Fig. 1d; Table S4). Therefore, we concluded that B2-3

314 was the gene from $\mathrm{CHC543-5}$ that was causal for late blight resistance. Henceforth,

315 we will refer to gene B2-3 as Rpi-chc1.1 as it is the first Rpi-chc1 allele that is

316 identified in S. chacoense.

317

318 Identification of Rpi-chc1.1 allelic variants

319

320 In order to identify different Rpi-chc1.1 allelic variants, we pursued an allele mining

321 approach in several resistant and susceptible $S$. chacoense, $S$. berthaultii, $S$.

322 tarijense, and $S$. tuberosum accessions. Homologous sequences were amplified

323 using primers overlapping the start and stop codons of Rpi-chc1.1. The PCR

324 fragments of the expected $3.9 \mathrm{~kb}$ size were cloned and sequenced, resulting in the

325 identification of fifteen Rpi-chc1.1-like sequences. From the selected diploid

326 accessions one or two sequence variants were identified, suggesting that indeed Rpi-

327 chc1 alleles were mined rather than paralogs. Phylogenetic analysis of the

328 sequences showed strong sequence similarities among the alleles (94.6 - 100\%

329 identity). Even within this high identity range, the presence of four main clades was

330 revealed (Fig. 2a). In clade 1, the Rpi-chc1.1 allele was found, together with three

331 sequences from $S$. berthaultii that were (nearly) identical to each other, and a

332 sequence from $S$. tarijense. From clade 1, together with Rpi-chc1.1, we selected one 
333 sequence from $S$. berthaultii (94-2031) and the $S$. tarijense (TAR852-5) for

334 complementation analysis. Transformation of the corresponding genes to susceptible

335 Désirée plants showed that they provide resistance to $P$. infestans isolates 90128

336 and IPO-C, like Rpi-chc1.1 (Table S5). We therefore concluded that clade 1 contains

337 functional alleles of Rpi-chc1. The S. tarijense allele will be referred to as Rpi-tar1.1.

338 The $S$. berthaultii allele will be referred to as Rpi-ber1.1 which matches to the

339 previously described Rpi-ber and Rpi-ber1 genes that were derived from the same

340 accession (PI473331) at similar genetic positions (Rauscher et al., 2006; Tan et al.,

341 2010; Vossen et al., 2013).

343 The allele mining in accession CHC543-5 resulted not only in the re-identification of

344 the active Rpi-chc1.1 but also in the identification a presumed allelic variant, which

345 we will refer to as Rpi-chc1.2. To test if Rpi-chc1.1 and Rpi-chc1.2 were indeed

346 alleles of the same gene, we tested Rpi-chc1.2 specific markers in the recombinant

347 population 6750 (CHC543-5 x CHC544-5). We found a perfect repulsion between

348 Rpi-chc1.2 and Rpi-chc1.1, strongly suggesting that both genes are allelic variants

349 (Table S6). Additionally, this analysis proved that Rpi-chc1.2 does not cause

350 resistance against $P$. infestans 90128, even though Rpi-chc1.2 is expressed during

351 infection (Fig. S3f). The Rpi-chc1.2 protein sequence clusters in clade 2 together with

352 four identical sequences from $S$. berthaultii. Close to clade 2, we can observe clade

353 3, which consisted of a $S$. berthaultii, a $S$. tarijense and a $S$. tuberosum allele from

354 RH89-039-16, a diploid clone previously characterised as susceptible to $P$. infestans

355 (Vleeshouwers et al., 2011a). The clade 3 allele from $S$. tarijense contained an in

356 frame stop codon, making it unlikely that this allele is producing an active resistance

357 protein. Additionally, a fourth clade contained only $S$. berthaultii alleles. The allelic

358 variants were numbered according to the clade in which they were found (i.e. Rpi-

359 ber1.1 from clade 1 and Rpi-ber1.2 from clade 2, etc.) followed by an extension to

360 indicate the genotype from which the allele was derived.

361

362 The mined Rpi-chc1.1 variants contained between 1296 to 1303 amino acids (Fig.

363 2b; Fig. S4) and the encoded proteins belong to the CNL-16 immune receptor family

364 (Witek et al. 2016). Their CC domains contain the $\mathrm{N}$ terminal MADA motif, 4

365 predicted $\alpha$-helices and the typical hhGRExE, but the distinctive EDVID motif was

366 less conserved. The NB domain contains the characteristic P-loop or Kinase 1a 
367 domain, the VYND motif, Kinase 2 domain or Walker B, and the Kinase 3a or RNBS-

368 B. The ARC1 domain contains the RNBS-C, the Motif 3 and the GLPL motif; and the

369 ARC2 contains the Motif 2, the RNBS-D and two copies of the MHDL motif. The LRR

370 domain consists of 29 imperfect repeats. Both LRR3 and LRR4 contain a central

371 VLDL motif which is conserved in the third LRR of most functional NLRs.

372

373 Rpi-chc1.1 recognises the RXLR PexRD12 effector family from $P$. 374 infestans

375

376

377

378

379

380

381

382

383

384

385

386

387

388

389

390

391

392

393

394

395

396

397

398

399

To understand the resistance mechanism of the S. chacoense CHC543-5 accession, we searched for the effector recognised by Rpi-chc1.1. A collection of ninety $P$. infestans extracellular (Pex) proteins in a PVX agroinfectious vector, of which 54 contained the RXLR-DEER motif (PexRD), was screened. Individual clones from the Pex collection were co-agroinfiltrated with Rpi-chc1.1 in N. benthamiana leaves. As a positive control, we used a mix of the R3a/AVR3a $R$ gene effector pair which is known to trigger a strong hypersensitive response (HR) in $N$. benthamiana leaves. Only two effectors from the Pex collection were able to trigger an Rpi-chc1.1 dependent HR, PexRD12-1 and PexRD12-2 (PITG_16233 and PITG_16240, respectively) (Fig. 3a). Neither the inactive paralogs B2-1 and B2-2 nor R3a produced an HR upon co-agroinfiltration with PexRD12. These results showed that PexRD12 is specifically recognised by Rpi-chc1.1. We could further confirm this finding using transgenic Désirée potato plants that were transformed with Rpi-chc1.1. About half of this transgenic population showed late blight resistance while the other half was susceptible, probably due to impaired transgene expression. Interestingly, the plants that showed late blight resistance also showed PexRD12 recognition, while the susceptible transgenic plants did not show any response upon PexRD12 agroinfiltration (Table S7). We sought for further evidence that PexRD12 was indeed causing a-virulence on Rpi-chc1.1 expressing plants. In a field trial with natural infection, we found isolates that were virulent on plants containing Rpi-chc1.1. The infected material was collected and used for gene expression analysis via RT-qPCR. The expression of PexRD12 was significantly reduced in the Rpi-chc1.1 resistance breaking isolates, while other effectors such as AVRsto1 were normally expressed. Reciprocally, we found that Rpi-sto1 breaking isolates still expressed PexRD12 
400 normally (Fig. 3b). Altogether, these results suggest that PexRD12 corresponds to

\section{AVRchc1.1.}

\section{2}

The PexRD12/31 superfamily is a complex $P$. infestans RXLR effector family

405 Using Blast analyses of the T30-4 proteome, we found 9 homologs of PexRD12 in 406 the $P$. infestans T30-4 genome. Additionally, we found that PexRD12 proteins had strong homology with 9 members of the PexRD31 family and two additional, more distantly related sequences (Table S3). These 20 effectors are encoded by clusters of paralogs mainly in three supercontigs (Fig. S5) and will henceforth be referred to as the PexRD12/31 superfamily (see also Petre et al., 2020). All PexRD12/31 effectors are small proteins that include a signal peptide in the $\mathrm{N}$-terminus, an effector domain in the C-terminus, and the conserved RXLR and EER motifs in the centre; except for PITG_16243 and PITG_09577 which contain an RXXR-EER and RXXLR-EER motifs, respectively (Fig. 4a).

The alignment of the protein sequences and the phylogenetic analysis of the

417 PexRD12/31 superfamily members resulted in five main clades (Fig. 4a). Two highly homologous clades can be distinguished to form the PexRD12 family, PexRD12-A1 and PexRD12-A2. The clade PexRD12-A2 also includes truncated versions which partly or completely miss the effector domain. In addition, two related clades constitute the PexRD31 family, PexRD31-B and PexRD31-C. Additionally, PITG_16428 and PITG_09577 were much less related and are together referred to as PexRD12/31 group D.

To determine the degree to which PexRD12/31 members are expressed in planta, as observed for other AVR effectors of $P$. infestans (Vleeshouwers et al., 2011b; Rietman et al., 2012), we tested their expression during infection with quantitative PCR on cDNA using clade $A, B$ and $C$ specific primers. The relative expression was calculated and normalised for the relative amount of $P$. infestans. Three different $P$. infestans isolates were evaluated at different time points after inoculation of different susceptible potato genotypes (Fig. S3a-d). In all the tested genotypes, PexRD12 432 showed the highest relative expression. In two isolates a maximum expression was 
433 found from 4 to 24 hours after inoculation and expression remained high till after 48

434 hours in all four isolates. The PexRD31-B effectors were expressed in 2 isolates but

435 were rapidly downregulated in the first hours after inoculation with hardly any

436 expression left. The expression of PexRD31-C was mostly undetectable along the

437 inoculation time course.

438

439 Rpi-chc1.2 recognises the RXLR PexRD31 effector family from $P$. 440 infestans

441

442

443

444

445

446

447

448

449

450

451

452

453

454

455

456

457

458

459

460

461

462

463

464

465

In order to describe the spectrum of effector recognition by different Rpi-chc1 alleles, several representatives from each clade were selected and co-agroinfiltrated with different PexRD12/31 members in N. benthamiana. Rpi-chc1.1_543-5 and Rpiber1.1_94-2031-01 from clade 1, Rpi-chc1.2_543-5 and Rpi-ber1.2_493-7 from clade 2, and rpi-tub1-RH89-039-16 from clade 3 were selected. As a representation from each of the clades of the PexRD12/31 effector superfamily, we selected: PITG_16245 (PexRD12-A1), PITG_20934 (PexRD12-A2), PITG_16235 (PexRD31B), and PITG_23069 (PexRD31-C). The different Rpi-chc1 alleles were coagroinfiltrated with the PexRD12/31 effectors in $N$. benthamiana leaves. Three days after agroinfiltration, we observed that the members from clade 1, Rpi-chc1.1 and Rpi-ber1.1, specifically recognised both PexRD12-A1 and PexRD12-A2 effectors (Fig. 4b). This result showed that Rpi-chc1.1 and Rpi-ber1.1 recognise multiple members of the PexRD12 family, suggesting that AVRchc1.1 is encoded by multiple redundant paralogs. On the other hand, Rpi-chc1.2 and Rpi-ber1.2 from clade 2, specifically recognised both PexRD31-B and PexRD31-C effectors (Fig. 4b). This suggests that multiple PexRD31 paralogs correspond to AVRchc1.2. The selected allele from clade 3, rpi-tub1.3_RH89-039-16, was not able to recognize any of the PexRD12/31 members (Fig. 4b), showing that clade 3 encodes functionally more distant receptors, and is in agreement with the known susceptibility of RH89-039-16 to P. infestans (Vleeshouwers et al., 2008).

\section{The LRR domain of the Rpi-chc1 variants determines the PexRD12/31 effector recognition specificity}


466 Since the allelic variants of Rpi-chc1 could be divided into three activity groups, while

467 having an amino acid identity up to $96 \%$, they provide ideal tools to investigate the

468 Rpi-chc1 mechanism of recognition. Therefore, we performed progressive exchanges

469 of the different receptor domains. The chimeric receptors were co-agroinfiltrated with

470 the PexRD12/31 effectors in $N$. benthamiana leaves to evaluate their recognition 471 specificity. First, we selected Rpi-chc1.2 and rpi-tub1.3_RH89-039-16 as

472 representatives of clade $B$ and clade $C$, respectively. When aligning the protein 473 sequences, 54 single amino acid polymorphisms (SAPs) were found and most of 474 them were located in the LRR domain (Fig. 5a). As previously mentioned, Rpi-chc1.2 475 specifically recognises PexRD31-B and PexRD31-C, while rpi-tub1.3_RH89-039-16 476 does not recognise any of the PexRD12/31 effectors. When the complete rpi-

477 tub1.3_RH89-039-16 LRR domain was exchanged for the Rpi-chc1.2 LRR, the 478 chimeric receptor $\mathrm{RH}:: \mathrm{C} 2$ 2-29 was able to recognise both PexRD31-B and 479 PexRD31-C. Reciprocally, the exchange of the Rpi-chc1.2 LRR for the rpi480 tub1.3_RH89-039-16 in $\mathrm{C} 2:: \mathrm{RH}$ 2-29, led to the inability to recognise any of the 481 PexRD12/31 effectors. This result demonstrates the importance of the LRR domain 482 during the AVRchc1.2 recognition. Additional domain exchanges were performed in 483 order to identify the essential LRR repeats for the effector recognition. The required 484 LRR repeats for the AVRchc1.2 recognition could be narrowed down with the 485 construct $\mathrm{RH}:: \mathrm{C2}_{2}$ 14-19 to nine amino acid polymorphisms (Fig. 5a). Due to the 486 absence of polymorphisms in the LRR repeats 14 and 15 , we can conclude that only 487 LRR repeats from 16 to 19 are required to activate the rpi-tub1.3_RH89-039-16 allele 488 to recognise AVRchc1.2. Interestingly, the majority of these nine amino acid 489 polymorphisms are particularly situated in the solvent exposed domain ( $x x L x L x x x x)$ of 490 every LRR repeat. The exchange of any of the solvent exposed residues for the 491 residues present in the inactive allele led to the partial or complete loss of effector 492 recognition (Fig. 5b).

493

494 To understand the difference in effector recognition specificity between Rpi-chc1.1 495 and Rpi-chc1.2, and to explore the possibility to combine both recognitions in one 496 receptor, we performed a similar progressive domain exchange approach between 497 Rpi-chc1.1 and Rpi-chc1.2 (Fig. 6). The exchange of the LRR domain in the chimeric 498 receptors $\mathrm{C} 1:: \mathrm{C} 2 \_8-29$ and $\mathrm{C} 2:: \mathrm{C} 1 \_8-29$ led to a shift in effector recognition, from 499 AVRchc1.1 to AVRchc1.2. Further exchanges revealed that the LRR repeats 14 to 23 
500 from Rpi-chc1.2 led to an opposite effector recognition pattern as the chimeric

501 receptor C1::C2_14-23 was able to only recognise AVRchc1.2. With reciprocal

502 domain exchanges of Rpi-chc1.1 into Rpi-chc1.2, we found that LRR repeats 8 to 29,

503 led to AVRchc1.1 recognition. In an attempt to further reduce the length of the

504 exchanged sequence, the recognition of AVRchc1.1 resulted in partial (C2::C1_8-25

505 and C2::C1_8-23) or complete (C2::C1_14-25 and C2::C1_14-23) loss of recognition.

506 Especially, when comparing the receptors C2::C1_8-29 and C2::C1_8-25, already

507 the modification of the last five SAPs led to the reduced recognition of AVRchc1.1.

508 But, apparently not only the last LRR repeats are involved in the effector recognition.

509 Also the first LRR repeats, from 8 to 14, are also important for AVRchc1.1 recognition

510 as C2::C1_8-25 was able to partially recognise AVRchc1.1, while C2::C1_14-25 did

511 not trigger any HR. We conclude that the LRR repeats 8 to 29 in Rpi-chc1.1 are

512 important for the AVRchc1.1 recognition, which overlaps with LRR repeats 16-19

513 from Rpi-chc1.2 which were required for AVRchc1.2 recognition.

\section{Discussion}

516 In this study, we identified Rpi-chc1.1 and 15 additional allelic variants from $S$.

517 berthaultii, S. tarijense and S. tuberosum. Phylogenetic analysis of the encoded 518 protein sequences revealed four clades. These four clades were not only supported 519 by sequence similarity but also by differences in effector and $P$. infestans recognition.

520 Clade 1 genes encode receptors that recognise PexRD12 effectors and includes the 521 active orthologs Rpi-chc1.1, Rpi-ber1.1 and Rpi-tar1.1 (Fig. 2, 3). Clade 2 receptors 522 could be distinguished by the recognition of the PexRD31 effectors (Fig. 4).

523 Receptors encoded by clades 3 and 4 do not recognise PexRD12/31 effectors and 524 no other activity has been found. Interestingly, clade 3 alleles are also present in 525 domesticated potato clones that are susceptible to late blight; e.g. RH89-039-16 (Fig.

526 2) and the varieties Colomba and Altus (unpublished data), implying that the encoded 527 receptors are not able to effectively provide resistance against $P$. infestans.

529 Rpi-ber1.1_94-2031-01 was derived from the same accession as the previously 530 described Rpi-ber (Rauscher et al., 2006; Vossen et al., 2009; Tan et al., 2010), and 531 Rpi-ber1 genes (Park et al., 2009). In these four studies, Rpi-ber and Rpi-ber1 532 mapped close to marker TG63 but slightly different genetic positions were reported. 
533 The population from Park et al. was quite small and a single recombination event

534 may have caused the deviating genetic distance. In the case of Tan et al., a single

535 mis-phenotyping could explain the mapping of Rpi-ber distal to TG63. We therefore

536 assume that Rpi-ber and Rpi-ber1 are the same genes and adopt the Rpi-ber1

537 naming as it is more consistent with current nomenclature for late blight resistance

538 genes. Rpi-ber2, as described by Park et al., was derived from the same accession

539 that was used in our allele mining studies (BER493). We could not find a clade 1 Rpi-

540 chc1 allele from the BER493 accessions, which supports the idea that a more

541 distantly related CNL16 member may be present that lacks sufficient match to the

542 primer sequences, explaining the Rpi-ber2 map position distal from TG63.

544 The presence of Rpi-chc1 alleles in $S$. tarijense and $S$. berthaultii suggests a 545 functional common ancestor existed before their speciation. However, it must be 546 noted that the geographic locations where the accessions were found are close to 547 each other in Bolivia. Since S. chacoense, S. tarijense and S. berthaultii are closely 548 related, the presence of functional Rpi-chc1 alleles in the three species might be a 549 result from a recent species intercrossing.

Sequence similarity among the studied allelic variants correlated with their

552 functionality, deduced by their ability to provide late blight resistance and $P$. infestans

553 effector recognition (Fig. 3, 4). This is not the first described case of $R$ gene allelic 554 variants across Solanum species. Rpi-blb1, Rpi-sto1 and Rpi-pta1, from the Mexican 555 species $S$. bulbocastanum, S. stoloniferum and $S$. papita are allelic variants that 556 recognise the same IpiO or PexRD6 P. infestans effector (Vleeshouwers et al., 557 2008). Among allelic variants of late blight resistance genes (i.e. Rpi-blb3 and Rpi558 hjt1 that recognise AVR2 effectors), overlapping recognition specificities have been 559 previously described (Champouret, 2010). Moreover, highly similar, but non-allelic $R$ 560 genes from the same CNL cluster had different recognition specificities, i.e. Rpi-vnt1, 561 Rpi-mcq1, R9a, Ph-3 (Smilde et al., 2005; Foster et al., 2009; Zhang et al., 2014; Jo et al., 2015). In the current report, we describe for the first time that allelic variants of 563 a late blight resistance gene show non-overlapping effector recognition specificities. 564 Remarkably, the recognized effectors belonged to the same effector family, which is an intriguing finding in the light of host pathogen co-evolution. 
567 When studying Rpi-chc1 protein domain structure, we identified most of the 568 conserved CNL motifs. Remarkably, the MADA motif (Adachi et al., 2019) was not 569 located downstream of the starting methionine, but downstream of the second 570 methionine in position 46 of the Rpi-chc1 protein. Further research is needed to show 571 if either or both methionines are used as translational start codons. Interestingly, we 572 recently cloned the functional late blight resistance gene from the late blight resistant 573 variety Carolus (Rpi-Carolus gene; Hamed Salehian, unpublished data). Rpi-Carolus 574 differed only at 7 amino acid positions from Rpi-ber1, but its $\mathrm{N}$-terminus was shorter 575 as a stop codon was present between the first two methionine codons. This strongly 576 suggests that translation in Rpi-Carolus starts from the second methionine while 577 retaining biological activity.

578

579 In contrast to the relatively conserved $\mathrm{N}$ termini of the proteins encoded by the Rpi580 chc1 alleles, most interallelic sequence variation localised to the Leucine Rich 581 Repeat regions. Through domain interchange between the rpi-tub1.3_RH89-039-16 582 and a Rpi-chc1.2 alleles and between Rpi-chc1.1 and Rpi-chc1.2, we discovered that 583 the LRR domain defines recognition specificity (Fig. 5, 6). Polymorphisms in the LRR 584 of some NLR receptors were previously shown to determine the effector recognition 585 specificity (Dodds et al., 2001; Shen et al., 2003; Catanzariti et al., 2010; Krasileva et 586 al., 2010; Ravensdale et al., 2012; Lindner et al. 2020). In one example, a domain exchange between $\mathrm{Rx} 1$ and Gpa2 converted the virus resistance into nematode resistance, and vice versa (Slootweg et al., 2017). The recognition of both nematode and virus could not be combined into one chimeric receptor, as we also observed with Rpi-chc1.1 and Rpi-chc1.2. The reason for this is the overlap between the LRRs involved in recognition.

592

593 Most of the amino acids in Rpi-chc1.2 that were required for AVRchc1.2 recognition 594 are located in the LRR solvent exposed motif (xxLxLXXXX) and modification of any of 595 the solvent exposed amino acids led to the partial or complete loss of PexRD31 596 recognition (Fig. 5b). The co-requirement of these solvent exposed amino acids 597 suggests that they are involved in recognition of a particular epitope. This 598 observation, combined with the observation of unequal distribution of SAPs, allow us 599 to hypothesise that Rpi-chc1 alleles evolved through insertion of a stretch of DNA into 600 the LRR domain rather than through accumulation of independent mutation. A similar 
601 model of evolution was recently proposed for allelic variants of Rpi-amr1 (Witek et al.,

602 2020). Such insertions may happen through unequal crossing-over with paralog

603 sequences or through retro-transposition. Interestingly, the evolution of integrated

604 domains in $R$ genes has been postulated to be caused by an unknown recombination

605 or transposon independent translocation mechanism (Bailey et al., 2018). The same

606 mechanism may be active in LRR exchange to evolve recognition of non-integrated

607 domains like guardees or decoys (Kourelis \& van der Hoorn, 2018) or direct effector

608 recognition.

609

610 Interestingly, some of the PexRD31 family members have been previously identified

611 as one of the most rapidly diversifying and fast evolving RXLR effectors in the T30-4

612 genome, with $\omega$ values higher than 1.55 (Haas et al., 2009). Additionally, several

613 members of the PexRD12/31 superfamily have recently been characterised to target

614 the host vesicle trafficking machinery by interacting with the vesicle associated

615 membrane protein 72 (VAMP72) family (Petre et al., 2020). Even though both

616 PexRD12 and PexRD31 have the same or functionally similar host targets, they are

617 differentially expressed during $P$. infestans infection. While PexRD12 is highly

618 expressed in all the tested isolates, PexRD31 is expressed at low levels after contact

619 with potato (Fig. S3). This would also explain why Rpi-chc1.2 is not able to provide

620 resistance against $P$. infestans, since most of the isolates have low expression levels

621 of AVRchc1.2. Consequently, clade A (PexRD12) may have evolved to avoid

622 detection by Rpi-chc1.2 while retaining its targeting of the vesicle trafficking

623 machinery.

624

625 Another step in the co-evolution between Rpi-chc1.1 and the PexRD12/31 family was

626 found by analysing the effector expression in plants expressing Rpi-chc1.1. The

627 isolates that overcome the Rpi-chc1.1 resistance no longer express PexRD12, while

628 the expression in untransformed Désirée plants was normal and comparable to the 629 expression of AVRsto1 (Fig. 3b). Similarly, evasion of recognition through

630 transcriptional suppression, was previously observed in plants expressing Rpi-vnt1

631 infected with $P$. infestans (Pel, 2010). Once more, we confirmed the plasticity of the

$632 P$. infestans effector secretion and the fast evolution capacity of some aggressive

633 isolates to break down single Rpi resistances. 
635 The introgression of single $R$ genes is driving $P$. infestans to evolve and evade

636 recognition. In order to durably deploy late blight resistance in agriculture, we need

637 novel strategies informed by knowledge of disease resistance genes in varieties, their

638 recognition specificities and the presence of the cognate effectors in the pathogen

639 populations. Virulence information from the field must be rapidly translated to

640 decision support systems (DSS) for the risk prediction and calculation of biocide

641 spraying intervals. Additionally, DSS can be used to determine $R$ gene composition

642 of (novel) varieties to be deployed in the next season. To meet these requirements,

643 novel breeding strategies are needed to rapidly tailor the $R$ gene contents of the

644 potato varieties to the pathogen populations. In current breeding schemes it takes

645 10-15 years to select a late blight resistant potato variety. Moreover, susceptible 646 varieties with dominant market shares will not be easy to replace. A system of

647 varieties with flexible $R$ gene content is needed. In other crops this has been 648 accomplished through F1 hybrid varieties. In potato, this route has only recently been 649 opened (Su et al., 2020) and no hybrid potato varieties have reached the market yet. 650 Proof of principle for flexible late blight resistance varieties produced through 651 cisgenesis was provided several years ago (Haverkort et al., 2016). Unfortunately, 652 the EU legislation does not distinguish between cisgenic and transgenic products, 653 making it impossible to market cisgenic varieties. Now, novel gene editing tools have 654 become available, and legislation for their application in agriculture is still under 655 debate. Knowledge as obtained in this study is essential to pursue such applications. 656 We now know how inactive resistance genes from susceptible varieties could be 657 repaired by replacing minimal fragments with the corresponding fragments of alleles 658 from wild relatives. This would provide an unprecedented accuracy and speed which 659 is not in introgression breeding.

660

661

662

663 The first part of this research was performed in the DuRPh project, funded by the 664 Ministry of Agriculture, Nature and Food Quality in the Netherlands. The second part 665 of the research was funded by the Ministry of Infrastructure and Water Management 666 in the Netherlands through the NWO-TTW program Biotechnology and Safety 667 (projectnumber 15815). We thank Jan de Boer and Adillah Tan for advice in selecting 668 BAC clones and marker sequences from chr10. Evert Jacobsen and Clemens van 
669 der Wiel are thanked for their advice about regulation and safety aspects of plant

670 biotechnology. Sidrat Abdullah is thanked for testing late blight resistance of

671 transgenic potato plants harbouring Rpi-chc1 allelic variants. Last but not least, we

672 thank Marjan Bergervoet, Gert van Arkel, Koen Pelgrom, Dirk Jan Huigen and Isolde

673 Pereira for plant transformations, molecular biology assistance and plant

674 maintenance.

675

676 Data Availability

677 All described sequences have been submitted top GenBank.

678

679

680

681 JV planned and designed the research; DML, MN and LK performed the majority of

682 the experiments; SK provided the Pex-RD set; DML and JV wrote the manuscript; RV

683 proofread the manuscript and provided the essential research environment. SA, HS

684 and KS contributed by mapping, cloning and characterization of Rpi-chc1 allelic

685 variants. RS, $A L$ and $A A H$ contributed through the identification of AVRchc1 and their

686 differential recognition specificities by Rpi-chc1 allelic variants.

687

688 References

Adachi H, Contreras MP, Harant A, Wu C, Derevnina L, Sakai T, Duggan C, Moratto

691 E, Bozkurt TO, Maqbool A, et al. 2019. An N-terminal motif in NLR immune receptors is

692 functionally conserved across distantly related plant species. eLife 8: e49956.

693 Aguilera-Galvez C, Champouret N, Rietman H, Lin X, Wouters D, Chu Z, Jones JDG,

694 Vossen JH, Visser RGF, Wolters PJ, et al. 2018. Two different R gene loci co-evolved with

695 Avr2 of Phytophthora infestans and confer distinct resistance specificities in potato. Studies in

696 Mycology 89: 105-115.

697 Bailey PC, Schudoma C, Jackson W, Baggs E, Dagdas G, Haerty W, Moscou M,

698 Krasileva KV. 2018. Dominant integration locus drives continuous diversification of plant

699 immune receptors with exogenous domain fusions. Genome Biology 19: 23.

700 Ballvora A, Ercolano MR, Weiss J, Meksem K, Bormann CA, Oberhagemann P,

701 Salamini F, Gebhardt C. 2002. The R1 gene for potato resistance to late blight

702 (Phytophthora infestans) belongs to the leucine zipper/NBS/LRR class of plant resistance

703 genes. The Plant Journal 30: 361-371.

704 Callaway E. 2013. Pathogen genome tracks Irish potato famine back to its roots. Nature:

705 nature.2013.13021. 
706 Catanzariti A-M, Dodds PN, Ve T, Kobe B, Ellis JG, Staskawicz BJ. 2010. The AvrM 707 Effector from Flax Rust Has a Structured C-Terminal Domain and Interacts Directly with the 708 M Resistance Protein. Molecular Plant-Microbe Interactions ${ }^{\circledR}$ 23: 49-57.

709 Caten CE, Jinks JL. 1968. Spontaneous variability of single isolates of Phytophthora 710 infestans. I. Cultural variation. Canadian Journal of Botany 46: 329-348.

711 Champouret N. 2010. Functional genomics of Phytophthora infestans effectors and Solanum 712 resistance genes. PhD thesis, Wageningen University. https://edepot.wur.nl/138174

713 Concordet J-P, Haeussler M. 2018. CRISPOR: intuitive guide selection for CRISPR/Cas9 714 genome editing experiments and screens. Nucleic Acids Research 46: W242-W245.

715 Dodds PN, Lawrence GJ, Ellis JG. 2001. Six Amino Acid Changes Confined to the 716 Leucine-Rich Repeat $\beta$-Strand/ $\beta$-Turn Motif Determine the Difference between the P and P2 717 Rust Resistance Specificities in Flax. The Plant Cell 13: 163-178.

718 Domazakis E, Lin X, Aguilera-Galvez C, Wouters D, Bijsterbosch G, Wolters PJ, 719 Vleeshouwers VGAA. 2017. Effectoromics-Based Identification of Cell Surface Receptors in 720 Potato. In: Shan L, He P, eds. Methods in Molecular Biology. Plant Pattern Recognition 721 Receptors. New York, NY: Springer New York, 337-353.

722 Engler C, Kandzia R, Marillonnet S. 2008. A One Pot, One Step, Precision Cloning 723 Method with High Throughput Capability (HA El-Shemy, Ed.). PLoS ONE 3: e3647.

724 Foster SJ, Park T-H, Pel M, Brigneti G, Śliwka J, Jagger L, van der Vossen E, Jones 725 JDG. 2009. Rpi-vnt1.1 a Tm-2 Homolog from Solanum venturii, Confers Resistance to 726 Potato Late Blight. Molecular Plant-Microbe Interactions ${ }^{\circledR}$ 22: 589-600.

727 Fry W. 2008. Phytophthora infestans $\square$ : the plant (and R gene) destroyer. Molecular Plant 728 Pathology 9: 385-402.

729 Goss EM, Press CM, Grünwald NJ. 2013. Evolution of RXLR-Class Effectors in the 730 Oomycete Plant Pathogen Phytophthora ramorum (A Palsson, Ed.). PLoS ONE 8: e79347.

731 Haas BJ, Kamoun S, Zody MC, Jiang RHY, Handsaker RE, Cano LM, Grabherr M, 732 Kodira CD, Raffaele S, Torto-Alalibo T, et al. 2009. Genome sequence and analysis of the 733 Irish potato famine pathogen Phytophthora infestans. Nature 461: 393-398.

734 Haverkort AJ, Boonekamp PM, Hutten R, Jacobsen E, Lotz LAP, Kessel GJT, Vossen 735 JH, Visser RGF. 2016. Durable Late Blight Resistance in Potato Through Dynamic Varieties 736 Obtained by Cisgenesis: Scientific and Societal Advances in the DuRPh Project. Potato 737 Research 59: 35-66.

738 Huang S, Van Der Vossen EAG, Kuang H, Vleeshouwers VGAA, Zhang N, Borm TJA, 739 Van Eck HJ, Baker B, Jacobsen E, Visser RGF. 2005. Comparative genomics enabled the 740 isolation of the R3a late blight resistance gene in potato: Cloning the potato late blight R3a 741 gene by synteny. The Plant Journal 42: 251-261.

742 Huang S, Vleeshouwers VGAA, Werij JS, Hutten RCB, van Eck HJ, Visser RGF, 743 Jacobsen E. 2004. The R3 Resistance to Phytophthora infestans in Potato is Conferred by 
744 Two Closely Linked R Genes with Distinct Specificities. Molecular Plant-Microbe 745 Interactions ${ }^{\circledR}$ 17: 428-435.

746 Jo K-R, Visser RGF, Jacobsen E, Vossen JH. 2015. Characterisation of the late blight 747 resistance in potato differential MaR9 reveals a qualitative resistance gene, R9a, residing in a 748 cluster of Tm-2 2 homologs on chromosome IX. Theoretical and Applied Genetics 128: 931749941.

750 Jo K-R, Zhu S, Bai Y, Hutten RCB, Kessel GJT, Vleeshouwers VGAA, Jacobsen E, 751 Visser RGF, Vossen JH. 2016. Problematic Crops: 1. Potatoes: Towards Sustainable Potato 752 Late Blight Resistance by Cisgenic $R$ Gene Pyramiding. In: Collinge DB, ed. Plant Pathogen 753 Resistance Biotechnology. Hoboken, NJ: John Wiley \& Sons, Inc, 171-191.

754 Jones DT, Taylor WR, Thornton JM. 1992. The rapid generation of mutation data matrices 755 from protein sequences. Bioinformatics 8: 275-282.

756 Jupe F, Witek K, Verweij W, Śliwka J, Pritchard L, Etherington GJ, Maclean D, Cock 757 PJ, Leggett RM, Bryan GJ, et al. 2013. Resistance gene enrichment sequencing (RenSeq) 758 enables reannotation of the NB-LRR gene family from sequenced plant genomes and rapid 759 mapping of resistance loci in segregating populations. The Plant Journal 76: 530-544.

760 Kourelis J, van der Hoorn RAL. 2018. Defended to the Nines: 25 Years of Resistance Gene 761 Cloning Identifies Nine Mechanisms for R Protein Function. The Plant Cell 30: 285-299.

762 Krasileva KV, Dahlbeck D, Staskawicz BJ. 2010. Activation of an Arabidopsis Resistance

763 Protein Is Specified by the in Planta Association of Its Leucine-Rich Repeat Domain with the

764 Cognate Oomycete Effector. The Plant Cell 22: 2444-2458.

765 Kumar S, Stecher G, Li M, Knyaz C, Tamura K. 2018. MEGA X: Molecular Evolutionary

766 Genetics Analysis across Computing Platforms (FU Battistuzzi, Ed.). Molecular Biology and

767 Evolution 35: 1547-1549.

768 Leister D. 2004. Tandem and segmental gene duplication and recombination in the evolution 769 of plant disease resistance genes. Trends in Genetics 20: 116-122.

770 Li G, Huang S, Guo X, Li Y, Yang Y, Guo Z, Kuang H, Rietman H, Bergervoet M, 771 Vleeshouwers VGGA, et al. 2011. Cloning and Characterization of R3b; Members of the R3 772 Superfamily of Late Blight Resistance Genes Show Sequence and Functional Divergence. 773 Molecular Plant-Microbe Interactions ${ }^{\circledR}$ 24: 1132-1142.

774

775

776

777

778

779

780

781

782

783
Lindner S, Keller B, Singh SP, Hasenkamp Z, Jung E, Müller MC, Bourras S and Keller B 2020. Single residues in the LRR domain of the wheat PM $3 A$ immune receptor can control the strength and the spectrum of the immune response. Plant Journal 104: 200-214.

Livak KJ, Schmittgen TD. 2001. Analysis of Relative Gene Expression Data Using RealTime Quantitative PCR and the 2- $\Delta \Delta \mathrm{CT}$ Method. Methods 25: 402-408.

Lokossou AA, Park T, van Arkel G, Arens M, Ruyter-Spira C, Morales J, Whisson SC, Birch PRJ, Visser RGF, Jacobsen E, et al. 2009. Exploiting Knowledge of R/Avr Genes to Rapidly Clone a New LZ-NBS-LRR Family of Late Blight Resistance Genes from Potato Linkage Group IV. Molecular Plant-Microbe Interactions® 22: 630-641. 
784 McBride KE, Summerfelt KR. 1990. Improved binary vectors for Agrobacterium-mediated 785 plant transformation. Plant Molecular Biology 14: 269-276.

786 Mcdowell JM, Simon SA. 2006. Recent insights into R gene evolution. Molecular Plant 787 Pathology 7: 437-448.

788 Park T-H, Foster S, Brigneti G, Jones JDG. 2009. Two distinct potato late blight resistance 789 genes from Solanum berthaultii are located on chromosome 10. Euphytica 165: 269-278.

790 Pel MA. 2010. Mapping, isolation and characterization of genes responsible for late blight 791 resistance in potato. $\mathrm{PhD}$ thesis, Wageningen University. https://edepot.wur.nl/138132

792 Pel MA, Foster SJ, Park T-H, Rietman H, van Arkel G, Jones JDG, Van Eck HJ, 793 Jacobsen E, Visser RGF, Van der Vossen EAG. 2009. Mapping and Cloning of Late Blight 794 Resistance Genes from Solanum venturii Using an Interspecific Candidate Gene Approach. 795 Molecular Plant-Microbe Interactions ${ }^{\circledR}$ 22: 601-615.

796 Petre B, Contreras MP, Bozkurt TO, Schattat MH, Sklenar J, Schornack S, Abd-El797 Haliem A, Castells-Graells R, Lozano-Duran R, Dagdas YF, et al. 2020. Host-interactor 798 screens of Phytophthora infestans RXLR proteins reveal vesicle trafficking as a major 799 effector-targeted process. Plant Biology.

800 Rauscher GM, Smart CD, Simko I, Bonierbale M, Mayton H, Greenland A, Fry WE. 801 2006. Characterization and mapping of Rpi-ber, a novel potato late blight resistance gene 802 from Solanum berthaultii. Theoretical and Applied Genetics 112: 674-687.

803 Ravensdale M, Bernoux M, Ve T, Kobe B, Thrall PH, Ellis JG, Dodds PN. 2012. 804 Intramolecular Interaction Influences Binding of the Flax L5 and L6 Resistance Proteins to 805 their AvrL567 Ligands (J-R Xu, Ed.). PLoS Pathogens 8: e1003004.

806 Rietman H, Bijsterbosch G, Cano LM, Lee H-R, Vossen JH, Jacobsen E, Visser RGF, 807 Kamoun S, Vleeshouwers VGAA. 2012. Qualitative and Quantitative Late Blight Resistance 808 in the Potato Cultivar Sarpo Mira Is Determined by the Perception of Five Distinct RXLR 809 Effectors. Molecular Plant-Microbe Interactions ${ }^{\circledR}$ 25: 910-919.

810 Sharma SK, Bolser D, de Boer J, Sønderkær M, Amoros W, Carboni MF, D’Ambrosio 811 JM, de la Cruz G, Di Genova A, Douches DS, et al. 2013. Construction of Reference 812 Chromosome-Scale Pseudomolecules for Potato: Integrating the Potato Genome with Genetic 813 and Physical Maps. G3\&amp;\#58; Genes|Genomes|Genetics 3: 2031-2047.

814 Shen Q-H, Zhou F, Bieri S, Haizel T, Shirasu K, Schulze-Lefert P. 2003. Recognition 815 Specificity and RAR1/SGT1 Dependence in Barley Mla Disease Resistance Genes to the 816 Powdery Mildew Fungus. The Plant Cell 15: 732-744.

817 Sievers F, Wilm A, Dineen D, Gibson TJ, Karplus K, Li W, Lopez R, McWilliam H, 818 Remmert M, Söding J, et al. 2011. Fast, scalable generation of high $\square$ quality protein 819 multiple sequence alignments using Clustal Omega. Molecular Systems Biology 7: 539.

820 Slootweg E, Koropacka K, Roosien J, Dees R, Overmars H, Lankhorst RK, van Schaik 821 C, Pomp R, Bouwman L, Helder J, et al. 2017. Sequence Exchange between Homologous 822 NB-LRR Genes Converts Virus Resistance into Nematode Resistance, and Vice Versa. Plant 823 Physiology 175: 498-510. 
824 Smilde WD, Brigneti G, Jagger L, Perkins S, Jones JDG. 2005. Solanum mochiquense 825 chromosome IX carries a novel late blight resistance gene Rpi-moc1. Theoretical and Applied 826 Genetics 110: 252-258.

827 Song J, Bradeen JM, Naess SK, Raasch JA, Wielgus SM, Haberlach GT, Liu J, Kuang 828 H, Austin-Phillips S, Buell CR, et al. 2003. Gene RB cloned from Solanum bulbocastanum 829 confers broad spectrum resistance to potato late blight. Proceedings of the National Academy 830 of Sciences 100: 9128-9133.

831 Su Y, Viquez-Zamora M, den Uil D, Sinnige J, Kruyt H, Vossen J, Lindhout P, van 832 Heusden S. 2020. Introgression of Genes for Resistance against Phytophthora infestans in 833 Diploid Potato. American Journal of Potato Research 97: 33-42.

834 Tan MYA, Hutten RCB, Visser RGF, van Eck HJ. 2010. The effect of pyramiding 835 Phytophthora infestans resistance genes R Pi-mcd1 and R Pi-ber in potato. Theoretical and 836 Applied Genetics 121: 117-125.

837 The Potato Genome Sequencing Consortium. 2011. Genome sequence and analysis of the 838 tuber crop potato. Nature 475: 189-195.

839 Vleeshouwers VG, Finkers R, Budding D, Visser M, Jacobs MM, van Berloo R, Pel M, 840 Champouret N, Bakker E, Krenek P, et al. 2011a. SolRgene: an online database to explore 841 disease resistance genes in tuber-bearing Solanum species. BMC Plant Biology 11: 116.

842 Vleeshouwers VGAA, Raffaele S, Vossen JH, Champouret N, Oliva R, Segretin ME, 843 Rietman H, Cano LM, Lokossou A, Kessel G, et al. 2011b. Understanding and Exploiting 844 Late Blight Resistance in the Age of Effectors. Annual Review of Phytopathology 49: 507845531.

846 Vleeshouwers VGAA, Rietman H, Krenek P, Champouret N, Young C, Oh S-K, Wang 847 M, Bouwmeester K, Vosman B, Visser RGF, et al. 2008. Effector Genomics Accelerates 848 Discovery and Functional Profiling of Potato Disease Resistance and Phytophthora Infestans 849 Avirulence Genes (HA El-Shemy, Ed.). PLoS ONE 3: e2875.

850 Vossen JH, van Arkel G, Bergervoet M, Jo K-R, Jacobsen E, Visser RGF. 2016. The 851 Solanum demissum R8 late blight resistance gene is an Sw-5 homologue that has been 852 deployed worldwide in late blight resistant varieties. Theoretical and Applied Genetics 129: $853 \quad 1785-1796$.

854 Vossen JH, Dezhsetan S, Esselink D, Arens M, Sanz MJ, Verweij W, Verzaux E, van der 855 Linden C. 2013. Novel applications of motif-directed profiling to identify disease resistance 856 genes in plants. Plant Methods 9: 37.

857 Vossen JH, Nijenhuis M, Arens M, Van Der Vossen EAG, Jacobsen E, Visser RGF. 858 2009. Cloning and exploitation of a functional $R$ gene from Solanum chacoense. Patent 859 application WO2011034433 A1. Published by the world intellectual property organization 18 860 September 2009

861 van der Vossen EAG, Gros J, Sikkema A, Muskens M, Wouters D, Wolters P, Pereira A, 862 Allefs S. 2005. The Rpi-blb2 gene from Solanum bulbocastanum is an Mi-1 gene homolog 863 conferring broad-spectrum late blight resistance in potato: Isolation of the late blight 864 resistance gene Rpi-blb2. The Plant Journal 44: 208-222. 
865 van de Vossenberg BTLH, Prodhomme C, van Arkel G, van Gent-Pelzer MPE, 866 Bergervoet M, Brankovics B, Przetakiewicz J, Visser RGF, van der Lee TAJ, Vossen 867 JH. 2019. The Synchytrium endobioticum AvrSen1 Triggers a Hypersensitive Response in 868 Sen1 Potatoes While Natural Variants Evade Detection. Molecular Plant Microbe 869 Interactions 32: 1536-1546.

870 van der Vossen E, Sikkema A, Hekkert B te L, Gros J, Stevens P, Muskens M, Wouters 871 D, Pereira A, Stiekema W, Allefs S. 2003. An ancient R gene from the wild potato species 872 Solanum bulbocastanum confers broad-spectrum resistance to Phytophthora infestans in 873 cultivated potato and tomato. The Plant Journal 36: 867-882.

874 Weber E, Engler C, Gruetzner R, Werner S, Marillonnet S. 2011. A Modular Cloning 875 System for Standardized Assembly of Multigene Constructs (J Peccoud, Ed.). PLoS ONE 6: $876 \mathrm{e} 16765$.

877 Werner S, Engler C, Weber E, Gruetzner R, Marillonnet S. 2012. Fast track assembly of 878 multigene constructs using Golden Gate cloning and the MoClo system. Bioengineered 3: 3887943.

880 Witek K, Jupe F, Witek A. et al. (2016). Accelerated cloning of a potato late blight881 resistance gene using RenSeq and SMRT sequencing. Nature Biotechnology 34, 656-660

882

883 Witek K, Lin X, Karki HS, Jupe F, Witek AI, Steuernagel B, Stam R, van Oosterhout C, 884 Fairhead S, Cocker JM, et al. 2020. A complex resistance locus in Solanum americanum 885 recognizes a conserved Phytophthora effector. BioRXiv 2020.05.15.095497 doi: 886 https://doi.org/10.1101/2020.05.15.095497

887 Zhang C, Liu L, Wang X, Vossen J, Li G, Li T, Zheng Z, Gao J, Guo Y, Visser RGF, et 888 al. 2014. The $\mathrm{Ph}-3$ gene from Solanum pimpinellifolium encodes CC-NBS-LRR protein 889 conferring resistance to Phytophthora infestans. Theoretical and Applied Genetics 127: 13538901364.

891 Zhu S, Vossen JH, Bergervoet M, Nijenhuis M, Kodde L, Vleeshouwers VGGA, Visser 892 RGF, Jacobsen E. 2015. An updated conventional- and a novel GM potato late blight R gene 893 differential set for virulence monitoring of Phytophthora infestans . Euphytica 202: 219-234 
A

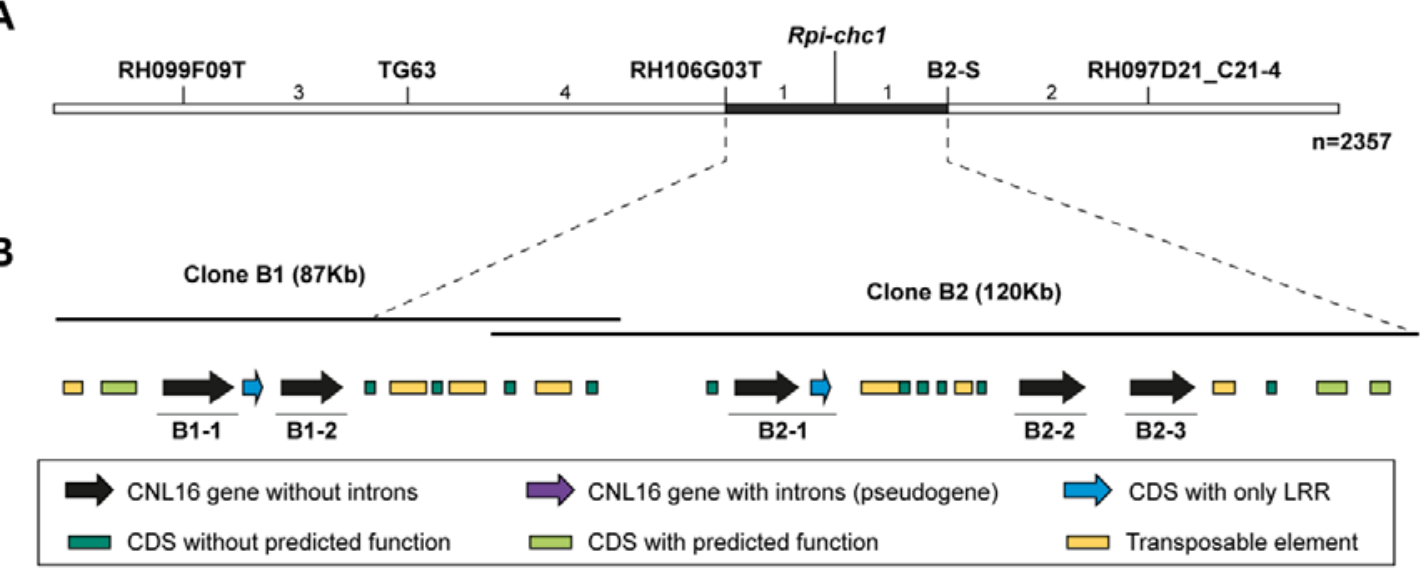

C

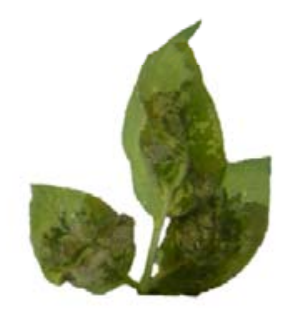

D

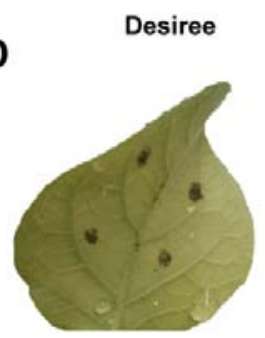

CHC543-5

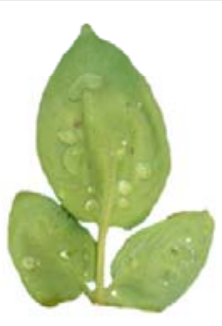

Desiree:Rpi-blb1

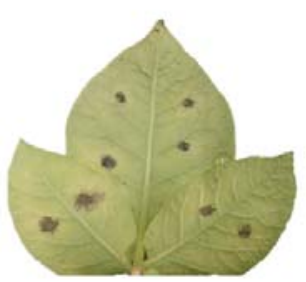

CHC543-5:CRISPR-1

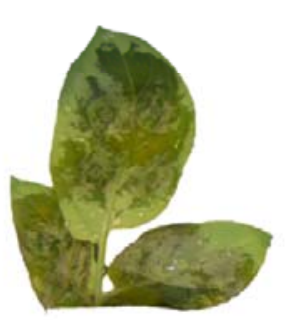

Desiree:B2-1

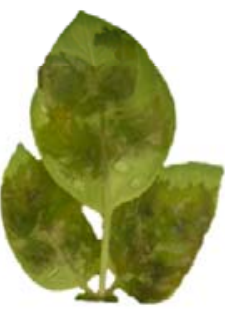

Desiree:B2-2

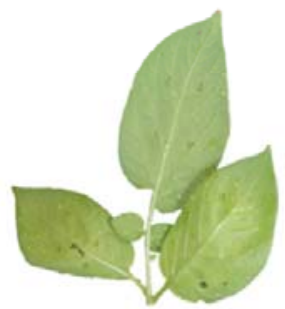

Desiree:B2-3

\section{Figures:}
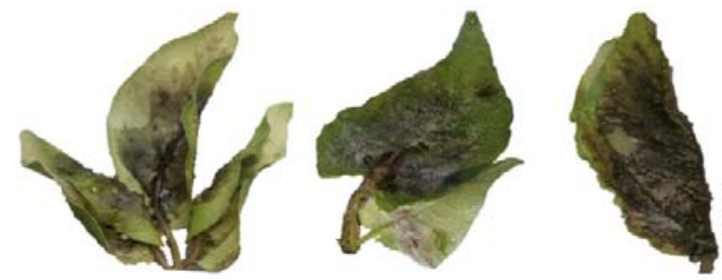

CHC543-5:CRISPR-21 CHC543-5:CRISPR-24 CHC543-5:CRISPR-26

896

897

898

899

900

901

902

903

904

905

906

907

908

909

910

911

912

Fig. 1. Map based cloning of Rpi-chc1.1.

(A) Genetic map of $P$. infestans (isolate 90128) resistance from CHC543-5. The number between the markers represents the number of recombinants found in a population derived from 2357 seedlings. Markers starting with $\mathrm{RH}$ were derived from BAC end sequences generated by PGSC. Marker B2-S represents the BAC end marker from clone 2. The black horizontal line represents the interval of Rpi-chc1.1. (B) Two BAC clones were isolated to generate the physical map. Annotation revealed the presence of NB-LRR genes, genes with or without predicted function and transposable elements. Three complete NB-LRR (B2-1, B2-2 and B2-3) genes between flanking markers RH106G03T and B2-S were selected as candidates. (C) The three candidates were stably transformed into the potato variety Desiree. After inoculation with isolate 90128 , only the candidate B2-3 was able to provide resistance. Untransformed Desiree and Desiree plants stably transformed with Rpiblb1 were used as negative and positive controls, respectively. (D) CRISPR-Cas9 constructs were designed to specifically target candidate B2-3 and stably transformed in the $S$. chacoense 543-5 resistant genotype. Transgenic plants with 
913 B2-3 knock-outs were susceptible to $P$. infestans 90128 and IPO-C isolates. 914 Transgenic plants without mutations in the B2-3 candidate and untransformed 915 CHC543-5 were used as a control.

A

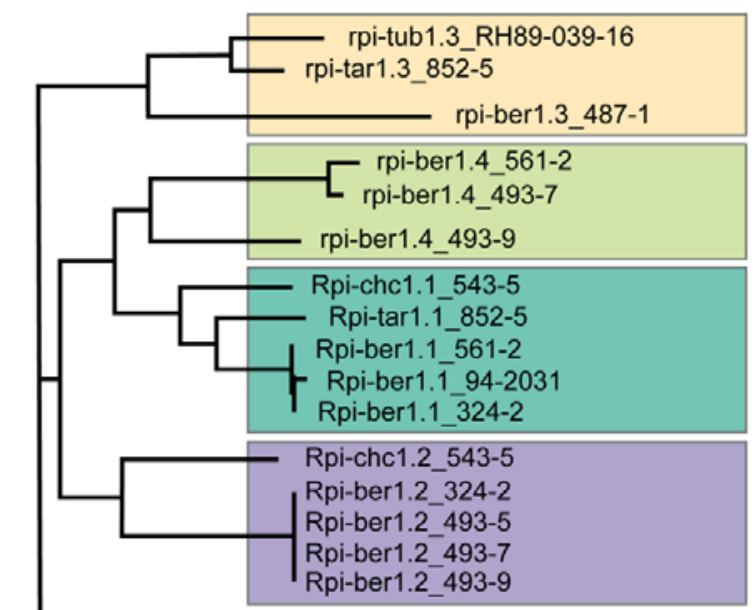

Clade 3

Clade 4

Clade 1

Clade 2

B

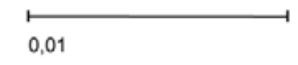

Soltu.DM.10G021850.1

916

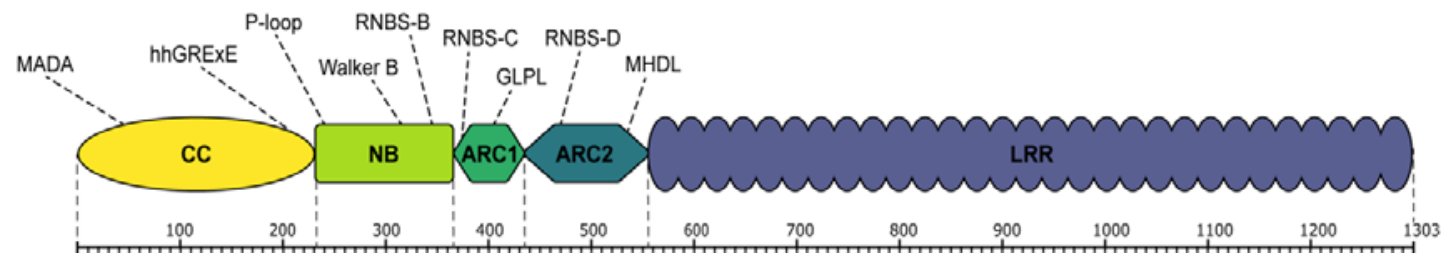

917 Fig. 2. Rpi-chc1 allele mining.

918 (A) Sixteen Rpi-chc1.1-like sequences were cloned from eleven different diploid

919 Solanum accessions. From seven accessions two variants were identified. From four

920 accessions only one variant was found, suggesting that the second allele did not

921 match the PCR primers. The phylogenetic analysis of the DNA sequences led to the

922 identification of four clades. The branch lengths represent the percentage of

923 phylogenetic distance. (B) The Rpi-chc1 alleles belong to the CNL immune receptor

924 family. Different motifs were found in the different receptor domains. The LRR

925

926 domain consists of 29 imperfect repeats. 
927

A

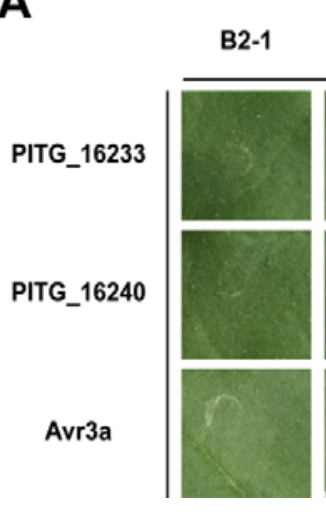

B2-2

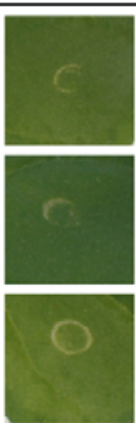

B2-3 (Rpi-chc1.1)

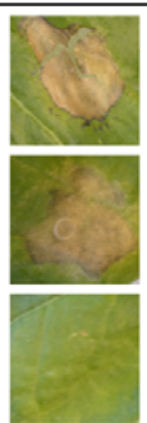

R3a

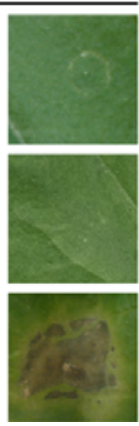

B

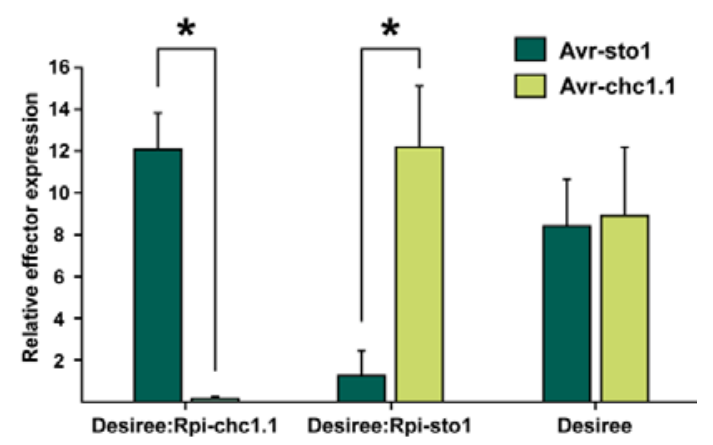

928

Fig. 3. The RXLR effector PexRD12 corresponds to Avrchc1.1.

929

(A) The three Rpi-chc1.1 candidates were co-infiltrated with the Pex effector

931 collection in N. benthamiana leaves to screen for Avrchc1.1. Rpi-chc1.1 induces cell

932 death when co-expressed with both PITG_16233 and PITG_16240, from the PexRD12 family. R3a and Avr3a were used as negative controls, a mix of R3a and Avr3a were used as a positive control. (B) Relative Avrchc1.1 and Avrsto1 effector expression in $P$. infestans field isolates collected from untransformed Desiree plants, and Desiree plants transformed with Rpi-chc1 (Desiree:Rpi-chc1.1), or Rpi-sto1

937 (Desiree:Rpi-sto1) (2013, Wageningen). Three independent samples were included 938 in the RT-qPCR experiment. Stars represent statistical difference in a two sample T-

939 test, $p<0.008$.

940 


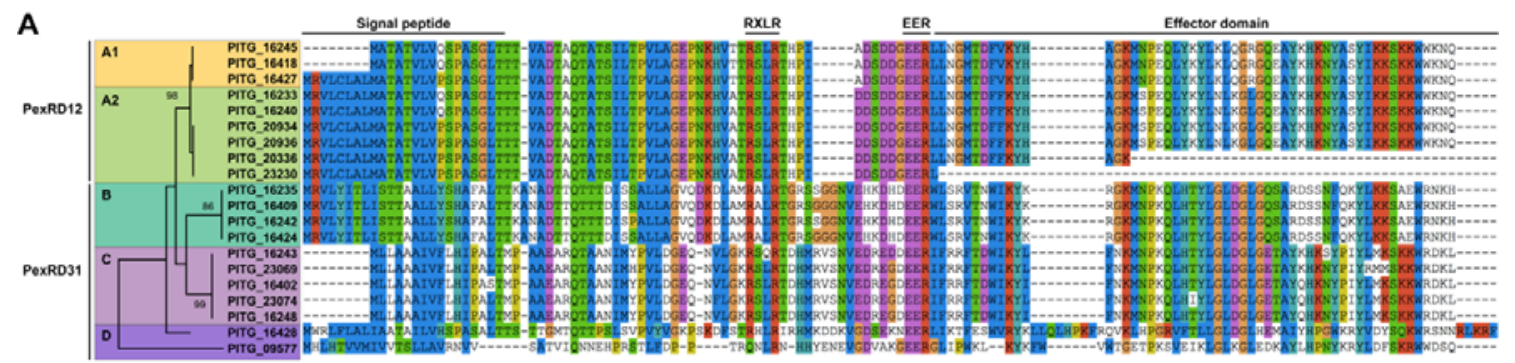

942

B

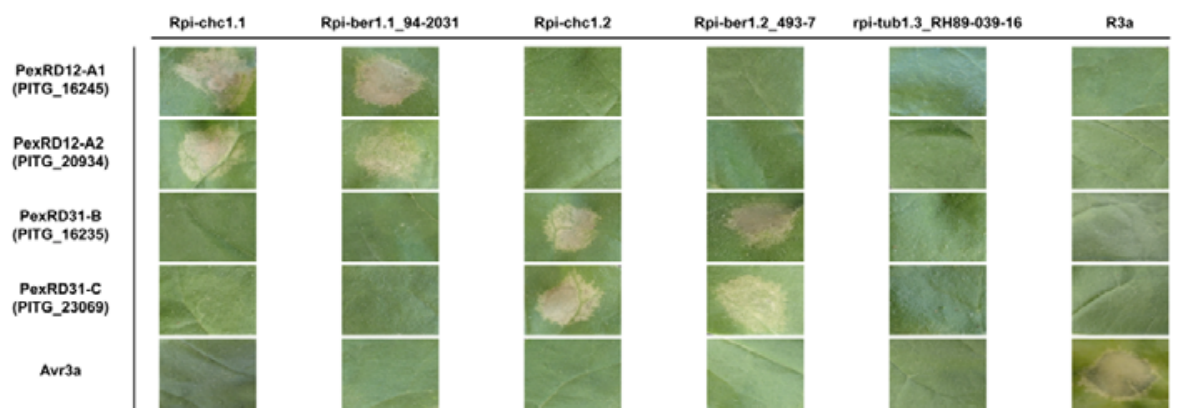

943 Fig. 4. Rpi-chc1 alleles show non-overlapping recognition of the PexRD12/31 effector 944 superfamily.

945 (A) The twenty members of the PexRD12/31 superfamily found in $P$. infestans isolate 946 T30-4. In the amino acid sequence we can distinguish a signal peptide in the $\mathrm{N}$ terminus, the conserved RXLR-EER motifs in the center, and the effector domain in the C- terminus. Some PexRD12/31 family members differed at the nucleotide level

950 but were identical at the protein level (PITG_16245 = PITG_16418; PITG_16233 =

951 PITG_16240; PITG_20934 = PITG_20936; PITG_16409 ${ }^{-}=$PITG_16424). The phylogenetic analysis of the complete protein sequences led to the identification of

952 five clades. This analysis was performed in MEGA $X$ by using the Maximum

953

954 Likelihood method based on the JTT matrix-based model. The tree with the highest log likelihood (-766) is shown. The bootstrapping values, which indicates the percentage of trees that had the particular branch, are shown in each branch. (B) Different Rpi-chc1 allelic variants were co-agroinfiltrated in $N$. benthamiana with a member from each PexRD12/31 clade. While variants from clade 1 recognize both PexRD12 A1 and A2 clades, Rpi-chc1 variants from clade 2 recognize PexRD31 B and C. A mix of R3a and Avr3a was used as a positive control. 


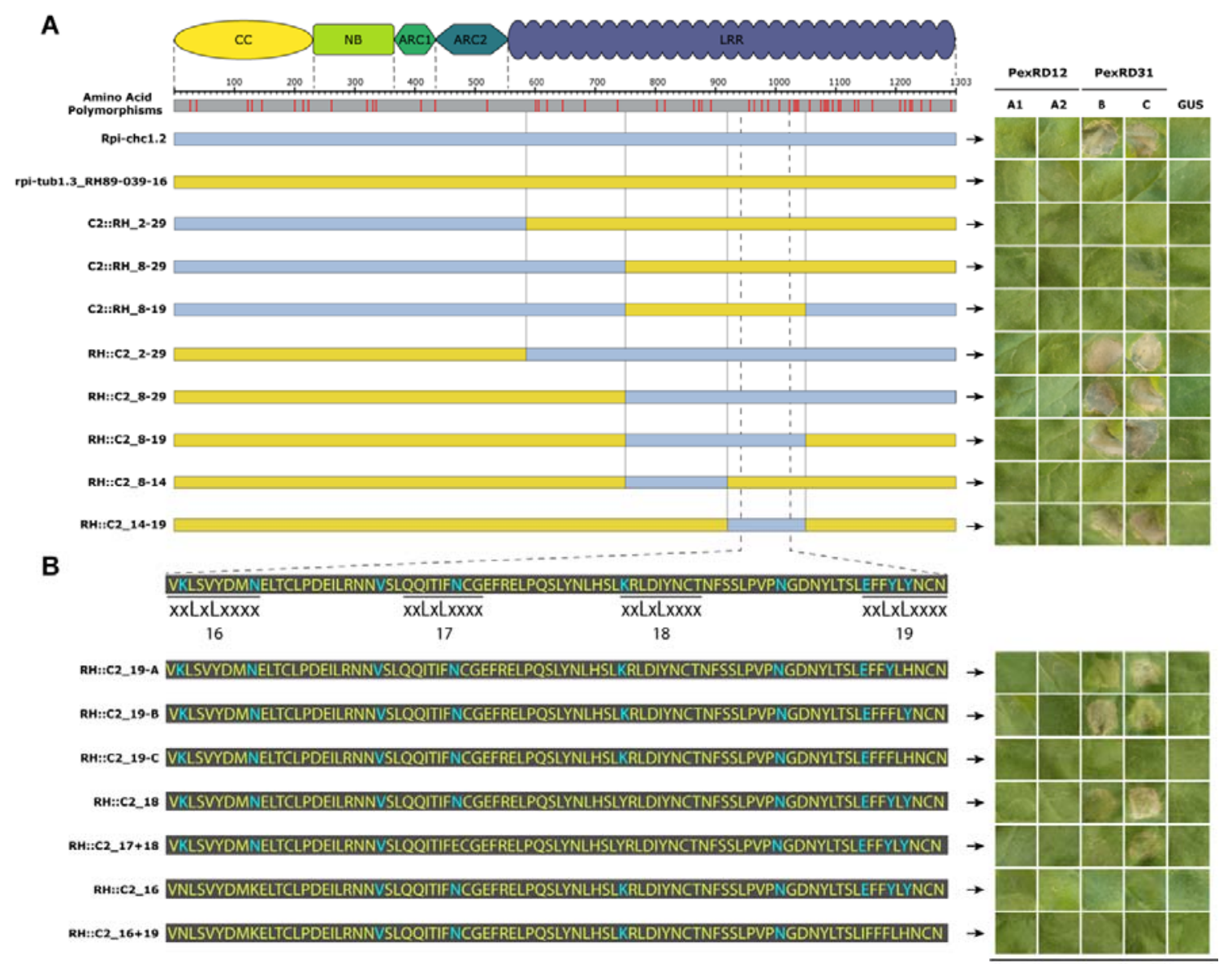

961

962

963

964

965

966

967

968

969

970

971

972

973

974

975

976

977

978

979

980

981

982

983

Fig. 5. Domain exchanges between rpi-tub1.3_RH89-039-16 and Rpi-chc1.2.

(A) The positions of SAPs and the corresponding protein domains are indicated on top. Rpi-chc1.2 and rpi-tub1.3_RH89-039-16, are represented as light blue and yellow bars, respectively. Below, the domain exchanges are shown. The chimeric constructs were co-agroinfiltrated with the PexRD12/31 effectors in N. benthamiana leaves. After 4 days, the HR that was visible and recorded. Experiments were repeated three times with 12 inoculation sites each time. A representative leaf for the response of each chimeric construct is shown in the right panel. GUS was used as a negative control. It is concluded that the exchange of the complete LRR domain led to recognition of PexRD31. With the final construct, $\mathrm{RH}:: \mathrm{C} 2$ 14-19, the exchange of only nine amino acids led to the activation of the rpi-tub1.3_RH89-036-16 protein. (B) Pinpointing of the amino acids involved in the Rpi-chc1.2 recognition specificity. SAPs are highlighted in blue font. Most of the SAPs are located in the solvent exposed xxLxLxxxx motif of the LRR 16-19. The chimeric constructs were coagroinfiltrated with the PexRD12/31 effectors in $N$. benthamiana leaves. A representative leaf for the response of each chimeric construct is shown in the right panel. Experiments were repeated three times with 12 inoculation sites each time. GUS was used as a negative control. The modification of any of the Rpi-chc1.2 solvent exposed specific amino acids (blue) for the corresponding amino acid present in rpi-tub1.3_RH89-039-16 (yellow), led to the partial or complete loss of effector recognition. 
984

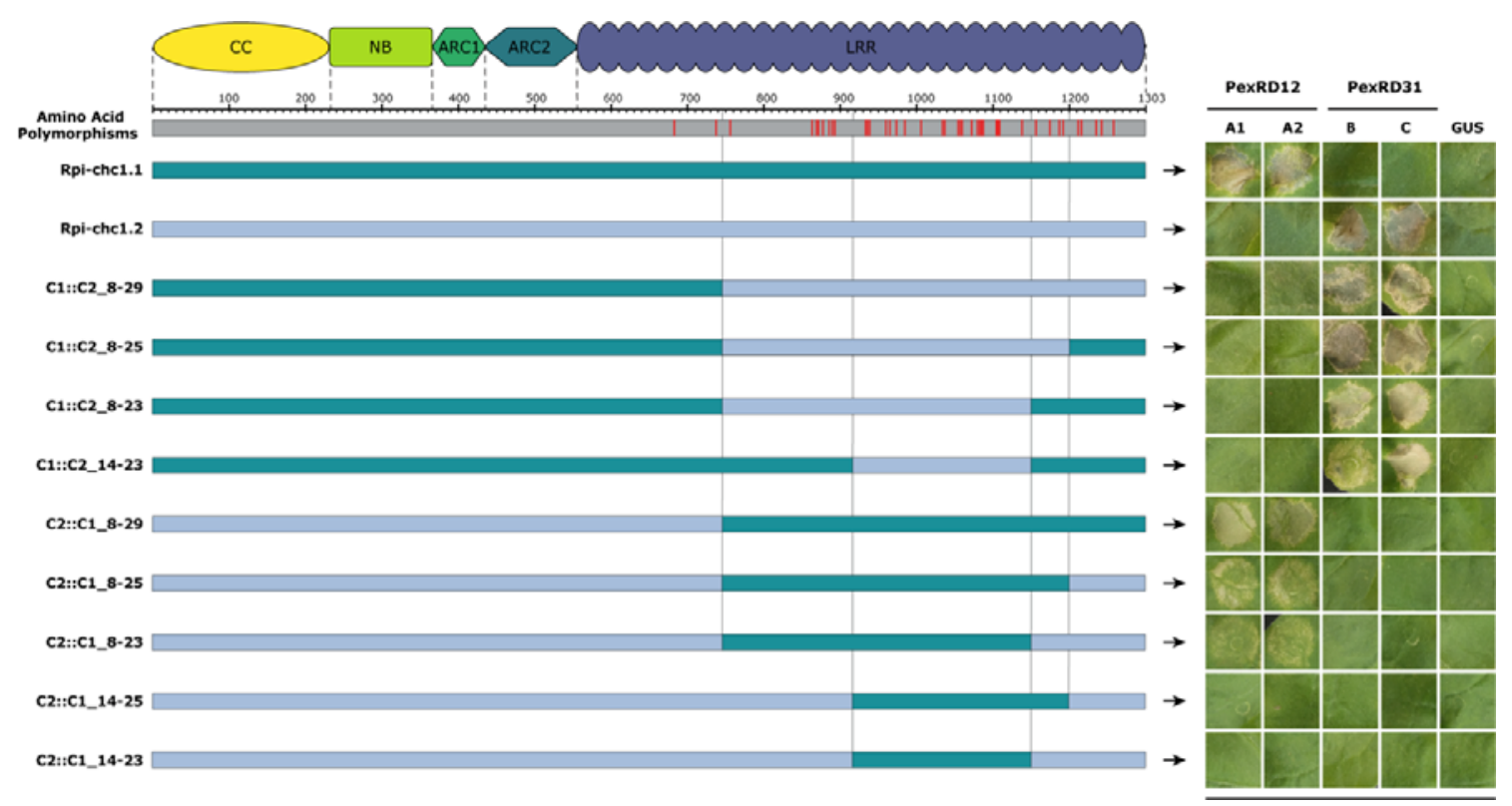

985

986

987

988

989

990

991

992

993

994

995

996

997

998
Fig. 6. The effector recognition specificity could be exchanged between Rpi-chc1.1 and Rpi-chc1.2.

The alignment of Rpi-chc1.1 and Rpi-chc1.2 shows that all the 41 amino acids polymorphisms (red bars) are located in the LRR domain. The chimeric constructs were co-agroinfiltrated with the PexRD12/31 effectors in $N$. benthamiana leaves. A representative leaf for the response of each chimeric construct is shown in the right panel. Experiments were repeated three times with 12 inoculation sites each time. GUS was used as a negative control. In the construct C1::C2_14-23, we could see that the LRR 16-19 reappear again as determining for the PexRD31 recognition. The required domain exchanges of the Rpi-chc1.1 LRR are more complex and encompasses almost the complete LRR. 


\section{Supporting Information:}

1000

1001 Fig. S1: pBINPLUS-PASSA-GG vector map.

1002 Fig. S2: Inoculation of P. infestans on N. benthamiana leaves agroinfiltrated with Rpi-chc1.1

1003 candidates.

1004 Fig. S3: Effector and R gene expression in potato leaves inoculated with P infestans..

1005 Fig. S4: Rpi-chc1.1 protein domain organization.

1006 Fig. S5: Localization of PexRD12/31 effectors in the P. infestans T30-4 contigs.

1007 Table S1: Accession numbers of Solanum genotypes and Rpi-chc1 sequences

1008 Table S2: Primers used in this study.

1009 Table S3: P. infestans effectors used in this study.

1010 Table S4: CRISPR-Cas9 targeting of Rpi-chc1.1.

1011 Table S5: Late blight resistance assessment of different Rpi-chc1 alleles.

1012 Table S6: Segregation of markers and late blight resistance of Rpi-chc1.1 and Rpi-chc1.2.

1013 Table S7: Functional expression of Rpi-chc1.1 in Desiree transgenic events correlates with

1014 responsiveness to PexRD12. 